\title{
Numerical calculation of the full two-loop electroweak corrections to muon $(g-2)$
}

\author{
Tadashi Ishikawa* \\ High Energy Accelerator Organization(KEK), 1-1 OHO Tsukuba Ibaraki 305-0801, Japan \\ Nobuya Nakazawa ${ }^{\dagger}$ \\ Department of Physics, Kogakuin University, Shinjuku, Tokyo 163-8677, Japan \\ Yoshiaki Yasui \\ Department of Management, Tokyo Management College, Ichikawa, Chiba 272-0001, Japan
}

(Received 19 October 2018; revised manuscript received 12 March 2019; published 12 April 2019)

\begin{abstract}
Numerical calculation of two-loop electroweak corrections to the muon anomalous magnetic moment $(g-2)$ is done based on on shell renormalization scheme (OS) and free quark model (FQM). The GRACE-FORM system is used to generate Feynman diagrams and corresponding amplitudes. Total 1780 two-loop diagrams and 70 one-loop diagrams composed of counterterms are calculated to get the renormalized quantity. As for the numerical calculation, we adopt the trapezoidal rule with double exponential method (DE). Linear extrapolation method (LE) is introduced to regularize UV- and IRdivergences and to get finite values. The reliability of our result is guaranteed by several conditions. The sum of one and two loop electroweak corrections in this renormalization scheme becomes $a_{\mu}^{\mathrm{EW}: \mathrm{OS}}[1+2$-loop $]=151.2( \pm 1.0) \times 10^{-11}$, where the error is due to the numerical integration and the uncertainty of input mass parameters and of the hadronic corrections to electroweak loops. By taking the hadronic corrections into account, we get $a_{\mu}^{\mathrm{EW}}[1+2$-loop $]=152.9( \pm 1.0) \times 10^{-11}$. It is in agreement with the previous works given in PDG [Phys. Rev. D 98, 030001 (2018)] within errors.
\end{abstract}

DOI: 10.1103/PhysRevD.99.073004

\section{INTRODUCTION}

In order to get a sign of beyond the standard model physics from high precision experimental data, we need higher order radiative corrections within the Standard Model (SM). For this purpose our group has been developing the automatic calculation system GRACE [1] since the late 1980s. The measurement of the muon anomalous magnetic moment $a_{\mu} \equiv(g-2) / 2$ is the one of the most precise experiments to check the SM. QED correction was calculated by T. Kinoshita et al. [2] up to tenth-order. The twoloop electroweak (ELWK) correction to $a_{\mu}$ was calculated approximately by Kukhto et al. [3] in 1992. Surprisingly, the two-loop correction is almost $20 \%$ of the one-loop correction [4-7]. We started to calculate the full two-loop

\footnotetext{
tishika@suchix.kek.jp

†nobuya@suchix.kek.jp

¥yasui@tokyo-keitan.ac.jp
}

Published by the American Physical Society under the terms of the Creative Commons Attribution 4.0 International license. Further distribution of this work must maintain attribution to the author(s) and the published article's title, journal citation, and DOI. Funded by SCOAP. corrections in 1995 and presented our formalism at Pisa conference [8]. We also showed that the two-loop QED value [9-12] was correctly reproduced within our general formalism. However, the number of diagrams is huge and the numerical integration requires the big CPU-power to achieve the required accuracy, we must wait until various environments are improved.

During these days, several groups did the approximate calculations [13-18] and the approximate value of the twoloop ELWK correction is widely accepted [19,20]. In 2001, BNL-Experiment 821 [21,22] announced that the precise experimental value deviates from that of SM around $(2.2-2.7) \sigma$ [23]. It brought much interest in the theoretical value. The main theoretical concern is now shifted to the hadronic contributions [24,25]. However, the discrepancy between the experimental value and the theoretical value is still large $\sim 3.5 \sigma[20,26]$. As new experiments at FNALE989 [27] will announce their first result in 2019 and J-PARC-E034 [28] is also planning the new experiment, we can expect to have new data soon.

\section{A. Perturbative numerical QFT}

Although the two-loop ELWK correction is almost established, we try to get the value without any approximation to 
confirm the validity of the earlier studies. ${ }^{1}$ This work is an important milestone to extend GRACE-system from oneloop to two-loop calculation. In ELWK theory, there are so many fields, mass parameters and complex couplings that it is hard to get reliable higher order corrections to physical quantities, in general. It is desirable to construct the framework to calculate these higher order corrections as automatically as possible. The key point is to perform Feynman integration numerically by using a sophisticated method with good convergence and a high power CPU machine. We propose to call such framework as perturbative numerical quantum field theory (PNQFT). The concepts of PNQFT are based on the following principles.

(a) It is essential to assume amplitudes as meromorphic functions of space time dimension $n$ for regularization and getting gauge invariant renormalized values of physical quantities.

(b) The source program for numerical integration is automatically generated by GRACE together with a symbolic manipulation system such as FORM [30].

(c) A high precision numerical integration method should be adopted.

(d) Linear extrapolation method (LE) (see Sec. III B) $[31,32]$ is crucial to extract both UV- and IR-divergences by taking advantage of the above analyticity. By the LE method, we can expand the amplitude in any order of $\varepsilon(=2-n / 2)$ (Laurent expansion), so that it is a powerful tool for higher order calculation.

(e) To guarantee the validity of the calculation, several conditions must be cleared. An example is the cancellation of nonlinear gauge (NLG) parameters.

(f) It is crucial to reduce human intervention to avoid careless mistakes. We must minimize the handmade operations necessary for getting the physical quantities.

The following calculation is based on these principles. In Sec. II and III, we briefly explain the flow and framework of our calculation. In Sec. IV, we touch on our method of numerical calculation. We emphasize that the linear extrapolation (LE) method is a simple and efficient method to regularize UV- and IR-divergences and also to get finite values. We also explain our consistency conditions to ensure the results. Some examples of calculations are explained. In Sec. V, we give our results on $a_{\mu}$. In the last section, we give some comments to make extensive progress. In Appendices, we explain the technical parts of our calculation.

\section{OUTLINE OF OUR FRAME WORK}

Our calculation is formulated under the following conditions.

(1) The calculation is done within SM.

\footnotetext{
${ }^{1}$ An intermediate stage of our calculation was reported in [29].
}

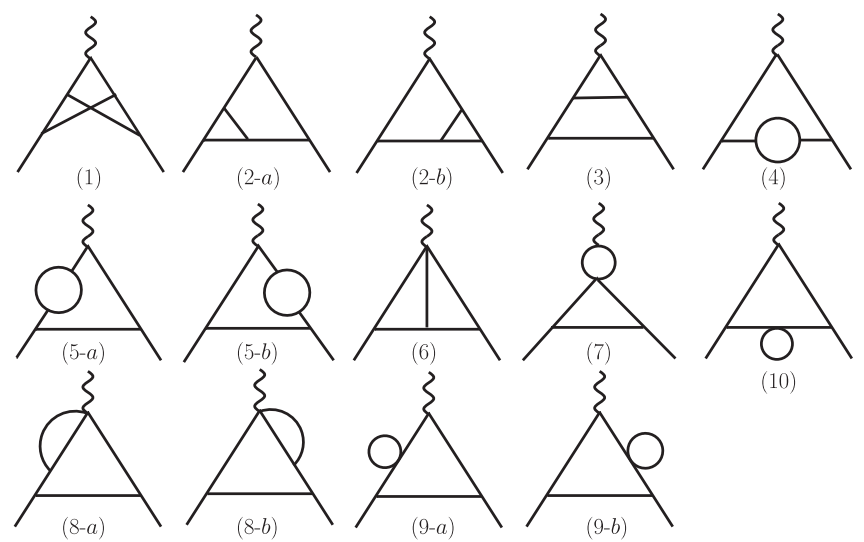

FIG. 1. Types of topology.

(2) On mass shell renormalization scheme (OS) is adopted [33-35]. We adopt $\alpha, M_{Z}, M_{W}, M_{H}$ and fermion masses as physical parameters. Weinberg angle, Higgs fermion coupling and other quantities are expressed by these parameters.

(3) Free quark model (FQM) is adopted and as for quarks, constituent masses are used.

(4) Nonlinear gauge formulation with a 't HooftFeynman propagator is adopted.

(5) Dimensional regularization is applied for both ultraviolet (UV)- and infrared (IR)-divergences.

(6) The linear extrapolation method (LE) is fully used for regularization and getting finite values.

Next, we briefly explain the flow of our calculation.

(1) GRACE system generates all the diagrams we need in SM, automatically [36]. There are 1780 two-loop diagrams ${ }^{2}$ and 70 one-loop diagrams composed of one-loop order counterterm (CT). Two-loop order CT is not necessary in our case, because $a_{\mu}$ is not related to the charge renormalization part.

(2) These 1780 diagrams are classified into 14 types of topology. Types of the topology are displayed in Fig. 1. Among these types, some of them give the same contribution because of symmetry. (an example: 5-a vs. 5-b) The diagrams including CT are classified into two types, namely, vertex and selfenergy types.

(3) GRACE system generates the amplitude of each diagram in accordance with Feynman rules for ELWK theory with NLG [35].

(4) In order to express the amplitude as a function of Feynman parameters used to combine denominators, we define the following quantities for each topology in advance $[37,38]$.

(i) Internal loop momentum flow $\left(\ell_{s}, \eta(s)\right)$ $(s=1,2)$

\footnotetext{
${ }^{2}$ There are 1678 diagrams in Feynman gauge and 102 extra diagrams specific to NLG.
} 
(ii) External momentum flow $\left(q_{j}\right) \quad(j=$ internal line number)

(iii) Kirchhoff's law of momentum conservation at each vertex

(iv) Feynman parameters are transformed to the integration variables in the interval $[0,1]$.

(5) Using these tools, contribution of each diagram to $a_{\mu}$ is expressed as a function of Feynman parameters, according to the formulas given in the next section. We make use of a symbolic manipulation system FORM exhaustively.

\section{LOGIC OF THE CALCULATION}

\section{A. Cvitanović-Kinoshita procedure}

In order to extract $a_{\mu}$ factor from muon vertex function, we adopt the Cvitanović-Kinoshita procedure [37,38]. We briefly explain the procedure in the case where there are six propagators. The starting formula is the two-loop muon vertex,

$$
\begin{aligned}
\Gamma_{\mu}= & \int \frac{d^{n} \ell_{1}}{i(2 \pi)^{n}} \frac{d^{n} \ell_{2}}{i(2 \pi)^{n}} \frac{F_{\mu}(D)}{\prod_{j}\left(p_{j}^{2}-m_{j}^{2}\right)} \\
= & \Gamma(6) \int \prod d z_{j} \delta\left(1-\sum_{j} z_{j}\right) \\
& \times \int \frac{d^{n} \ell_{1}}{i(2 \pi)^{n}} \frac{d^{n} \ell_{2}}{i(2 \pi)^{n}} \frac{F_{\mu}(D)}{\sum_{j} z_{j}\left(p_{j}^{2}-m_{j}^{2}\right)}
\end{aligned}
$$

where $p_{j}=\sum_{s=1}^{2} \eta_{s}(j) \ell_{s}+q_{j}$, is the momentum on the internal line $(j)$. The function $\eta_{s}(j)(= \pm 1,0)$ defines the weight of loop momentum $\ell_{s}$ on the internal line $(j)$. The $z_{j}$ 's are the Feynman parameters to combine six propagators. $F_{\mu}(D)$ is the numerator function and $\mu$ is the external photon polarization. Next we diagonalize the denominator function with respect to loop momenta $\ell_{1}, \ell_{2}$ and perform integration. The result is,

$$
\begin{aligned}
\Gamma_{\mu}= & \frac{1}{(4 \pi)^{n}} \int \prod d z_{j} \delta\left(1-\sum_{j} z_{j}\right) \frac{\Gamma(6-n)}{(\operatorname{det} \mathrm{U})^{n / 2}} \\
& \times F_{\mu}(D) \frac{1}{(V-i \epsilon)^{6-n}}, \\
U_{s, t}= & \sum_{j=1}^{6} z_{j} \eta_{s}(j) \eta_{t}(j) .
\end{aligned}
$$

Where $U$ is well known $2 \times 2$ matrix, composed of Feynman parameters $\left(z_{j}\right) . V\left(z_{j}, m_{j}, q_{j}\right)$ is the denominator function. The argument is easily extended to the case with five-propagators (diagrams with four-point coupling).

In order to generate the numerator function we use the following differential integral operator $D_{j}^{\mu}$.
$\frac{p_{j}^{\mu}}{\left(p_{j}^{2}-m_{j}^{2}\right)}=D_{j}^{\mu} \frac{1}{\left(p_{j}^{2}-m_{j}^{2}\right)}, \quad D_{j}^{\mu} \equiv \frac{1}{2} \int_{m_{j}^{2}}^{\infty} d m_{j}^{2} \frac{\partial}{\partial q_{j \mu}}$

The operator $D_{j}^{\mu}$ generates momentum $p_{j}^{\mu}$ on the internal line $(j)$. By operating $D_{j}^{\mu}$ to the denominator function $V$, we get the following expression.

$$
\begin{gathered}
D_{i}^{\mu} D_{j}^{\nu} \frac{1}{V^{m}}=\frac{Q_{i}^{\mu} Q_{j}^{\nu}}{V^{m}}+\left(-\frac{1}{2 \operatorname{det} \mathrm{U}}\right) \frac{g^{\mu \nu}}{(m-1)} \frac{B_{i j}}{V^{m-1}}, \\
Q_{j}^{\mu}=q_{j}^{\mu}-\frac{1}{\operatorname{det} U} \sum_{i=1}^{6} z_{i} B_{i j} q_{i}^{\mu}, \\
B_{i j}=\sum_{s, t} \eta_{s}(i) \eta_{t}(j) U_{s t}^{-1} \operatorname{det} U=B_{j i} .
\end{gathered}
$$

Using the above formulas, we can write down the numerator functions in terms of $B_{i j}$ algebraically. The equivalence of the above method and the well-known method of shifting loop momentum to diagonalize the denominator function is verified. The correspondence between two methods are symbolized as follows.

$$
\begin{aligned}
\ell^{0} & \rightarrow \frac{\left\{1, Q_{i}^{\mu}, Q_{i}^{\mu} Q_{j}^{\nu}, \cdots\right\}}{V^{m}}, \\
\ell_{i}^{\mu} \ell_{j}^{\nu} & \rightarrow\left(-\frac{1}{2 \operatorname{det} \mathrm{U}}\right) \frac{g^{\mu \nu}}{(m-1)} \frac{B_{i j}}{V^{m-1}} .
\end{aligned}
$$

The next step is to extract the $a_{\mu}$ factor by using projection operator, from the photon muon vertex $\Gamma_{\mu}$. The quantity $a_{\mu}$ is given as follows. ( $m_{0}=$ muon mass)

$$
\begin{aligned}
a_{\mu} & =\lim _{q^{2} \rightarrow 0} \frac{m_{0}}{p^{4} q^{2}} \operatorname{Tr}\left(\Gamma_{\mu} \operatorname{Proj}(\mu)\right) \\
\operatorname{Proj}(\mu)= & \frac{1}{4}\left(\not p-\frac{1}{2} \not q+m_{0}\right)\left\{\mathrm{m}_{0} \gamma_{\mu}(p \cdot p)-\left(m_{0}^{2}+\frac{q \cdot q}{2}\right) p_{\mu}\right\} \\
& \times\left(\not p+\frac{1}{2} \not q+\mathrm{m}_{0}\right),
\end{aligned}
$$

where we set momentum of incoming $\mu^{-}$, outgoing $\mu^{-}$, and incoming photon, as $(p-q / 2),(p+q / 2)$ and $q$, respectively. The final expression for numerical integration is summarized in the following formula.

$$
\begin{aligned}
F= & \frac{1}{(4 \pi)^{n}} \int \prod d z_{j} \delta\left(1-\sum_{j} z_{j}\right) \\
& \times\left[\frac{\Gamma(6-n) f_{0}}{(\operatorname{det} \mathrm{U})^{n / 2}(V-i \epsilon)^{6-n}}+\frac{\Gamma(5-n) f_{2}}{-2(\operatorname{det} \mathrm{U})^{n / 2+1}(V-i \epsilon)^{5-n}}\right] .
\end{aligned}
$$

The numerators $f_{0}, f_{2}$ represent the coefficient of $\ell^{0}, \ell^{2}$ term, respectively, after projection operator is applied. They are also the function of dimension $n$. 


\section{B. Regularization method}

Next step is the regularization of UV- and IR-divergences. By adopting $n$-dimensional regularization method, any integrand $F$ of Feynman parameter integration is regarded as a function of $\varepsilon=2-n / 2, F(\varepsilon)$. We adopt two methods for regularization.

\section{Linear extrapolation method}

The first one is very simple and powerful if the accuracy of numerical integration is sufficiently guaranteed. We call it the linear extrapolation method (LE). Just after the dimensional regularization method was introduced $[39,40]$, the analyticity with respect to $\varepsilon$ was discussed extensively. It is shown that the Feynman amplitude is a meromorphic function of $\varepsilon$ [39-41]. This is a key point to utilize the LE method to the Feynman amplitude. The following are the steps to get the divergent and finite terms.

(1) Calculate $G(\varepsilon)=\int F\left(z_{j}, \varepsilon\right) \prod d z_{j}$ for various values of $\varepsilon=\varepsilon(i) .(i=1,2, \ldots M)$.

(2) We set $\varepsilon(i)=1 / \alpha^{i+14}$ by taking relevant value $\alpha$.

(3) According to the analyticity, we can expand $G(\varepsilon(i))$ in Laurent series. In our case, it is evident that the expansion starts from $(1 / \varepsilon(i))$ because of the lack of two-loop counterterms. We truncate the series at $\mathrm{O}\left(\varepsilon(i)^{M-2}\right)$.

$$
\begin{aligned}
G(\varepsilon(i)) & =C_{-1} \frac{1}{\varepsilon(i)}+C_{0}+C_{1} \varepsilon(i)+\cdots+C_{M-2} \varepsilon(i)^{M-2} \\
& =\sum_{j=-1}^{M-2} C_{j}\{\varepsilon(i)\}^{j}(i=1,2, \ldots M) .
\end{aligned}
$$

The coefficients $C_{-1}$ and $C_{0}$ correspond to the divergent and finite parts, respectively. In the case $\varepsilon=2-n / 2, C_{-1}$ expresses the UV-divergent part and if we set $\varepsilon \rightarrow \varepsilon_{R}=(n / 2-4), C_{-1}$ represents the IR-divergent part.

(4) To get $\left\{C_{j}\right\}$, we multiply the inverse of $M \times M$ matrix $A$, whose element is $A(i, j)=\{\varepsilon(i)\}^{j}$, $(i=1, \cdots M, j=-1,0, \ldots, M-2), \quad$ to $\mathrm{M}$-component vector $G(\varepsilon(i))$.

$$
C_{j}=\sum_{i=1}^{M} A^{-1}(j, i) G(\varepsilon(i)), \quad(j=-1,0, \ldots, M-2) .
$$

(5) In order to improve the convergence, we set $M \sim 18$ and $\alpha \sim 1.1$ by trial and error. Examples setting these parameters are shown in [31,32]

(6) Various methods are known to extract $C_{-1}, C_{0}$ from $G(\varepsilon(i))$ [42], however, LE method is simple and appropriate in our case.
In order to get the reliable value of $C_{-1}, C_{0}$ up to 4 digits, we need the accuracy of the numerical integration at least 8 digits.

\section{Subtraction method}

To complement the above calculation, we also adopt the well known subtraction method to separate divergent part and finite part. We extract $1 / \varepsilon$ singularity from $G(\varepsilon)$ when one of Feynman parameters approaches $0,(x \rightarrow 0)$. The followings are the steps to extract the singularity.

(1) First we transform the Feynman parameters $\left(z_{1}, z_{2}, \ldots, z_{6}\right)$ into the appropriate $[0,1]$ variables $(x, y, u, v, w)$ depending on the topology. Key point is to factorize the function $\operatorname{det} U=x \times z(x, \cdots)$, where $z(0, \cdots) \neq 0$. Singular behavior $(1 / \varepsilon)$ comes from the factor $(\operatorname{det} U)^{n / 2}$ in Eq. (2)

(2) The following formula is effective to extract the factor $(1 / \varepsilon)$ for vertex type correction.

$$
\begin{aligned}
I= & \int_{0}^{1} x^{\varepsilon-1} F(x, \varepsilon) d x=\frac{1}{\varepsilon} F(0,0)+\frac{\partial F(0,0)}{\partial \varepsilon} \\
& +\int_{0}^{1} \frac{F(x, 0)-F(0,0)}{x} d x+O(\varepsilon) .
\end{aligned}
$$

(3) In the case where there is self-energy type diagram, the factor $x^{\varepsilon-2}$ appears in the head of integrant. If we expand it in $\varepsilon$ by using analytic continuation the following formula is obtained.

$$
\begin{aligned}
I= & \int_{0}^{1} x^{\varepsilon-2} F(x, \varepsilon) d x \\
= & \frac{1}{\varepsilon} \frac{\partial F(0,0)}{\partial x}+\frac{\partial F(0,0)}{\partial x}+\frac{\partial^{2} F(0,0)}{\partial \varepsilon \partial x}-F(1,0) \\
& +\int_{0}^{1} \frac{\partial F(x, 0) / \partial x-\partial F(0,0) / \partial x}{x} d x+O(\varepsilon) .
\end{aligned}
$$

We use this method partly to complement the LE method.

\section{Counterterms}

As for counterterms, GRACE has a library of renormalization constants at one-loop level based on OSrenormalization scheme. We make use of this library for 70-diagrams composed of counterterms. Generally speaking, it is necessary to expand one-loop renormalization constants up to order $\varepsilon=2-n / 2$ at two-loop level. However, the divergent part of diagrams composed of CT does not contribute to $a_{\mu}$, the $O(\varepsilon)$ term is unnecessary in our case. Here we comment on the wave function renormalization constant of Goldstone fields $\chi, \chi_{3}$. We keep the finite part of the constant in the form $(-1 / 2)\left\{d \Pi^{\chi \chi}\left(q^{2}\right) / d q^{2}\right\}$ at $q^{2}=M_{W}^{2}$. However, the final answer is independent of the finite part. For the 
renormalization of unphysical fields, the UV-divergent part is only useful to erase divergence.

\section{NUMERICAL CALCULATION}

\section{A. Double exponential method}

The final step to get the value $a_{\mu}$ is the numerical integration over Feynman parameters. We employ trapezoidal rule with double exponential (DE) transformation method [43]. It is also called as $\tan h-\sin h$ transformation method. It is very powerful if the integrand has singular behavior at the edge of the integration domain. Speed of convergence is accelerated by the DE transformation,

$$
I=\int_{0}^{1} d x f(x) \rightarrow x=\phi(t)=\frac{1}{2}\left\{1+\tanh \left(\frac{\pi}{2} \sinh (t)\right)\right\} .
$$

The maximum dimension of multiple integration is five. We apply DE method to any integration variable involved. As we need the accuracy greater than 8 digits to see the UV cancellation, the adaptive Monte Carlo method is not adopted in our two-loop calculation.

\section{B. Criterion to ensure the validity of the result}

In order to ensure the validity of our results, we impose several conditions given below.

(1) Well known QED two-loop value is reproduced up to 7 digits.

(2) UV-divergence is cancelled.

(3) IR-divergence is cancelled.

(4) The result is independent of nonlinear gauge parameters.

(5) In some cases (examples: topology 4,5-a,5-b,7, $9-1,9 \mathrm{~b}$ and 10), we can perform loop-integrations $\ell_{1}$ and $\ell_{2}$ successively. (We call it successive method.) We obtain the same value as the direct method previously shown.

In all these cases, if we have plural methods to evaluate, we compare the numerical values to ascertain the validity. We demonstrate how the conditions are cleared by showing the examples in the Appendices.

\section{Nonlinear gauge (NLG) parameter independence}

Originally nonlinear gauge was introduced to reduce the number of diagrams, particularly containing boson-boson couplings [44-49]. Here, we adopt NLG to check the validity of our calculation. The gauge fixing Lagrangian is constructed as,

$$
\mathscr{L}_{G F}=-\frac{1}{\xi_{W}} F^{+} F^{-}-\frac{1}{2 \xi_{Z}}\left(F^{Z}\right)^{2}-\frac{1}{\xi}\left(F^{A}\right)^{2}
$$

where

$$
\begin{aligned}
F^{ \pm}= & \left(\partial^{\mu} \mp i e \tilde{\alpha} A^{\mu} \mp i \frac{e c_{W}}{s_{W}} \tilde{\beta} Z^{\mu}\right) W_{\mu}^{ \pm} \\
& +\xi_{W}\left(M_{W} \chi^{ \pm}+\frac{e}{2 s_{W}} \tilde{\delta} H \chi^{ \pm} \pm i \frac{e}{2 s_{W}} \tilde{\kappa} \chi_{3} \chi^{ \pm}\right) \\
F^{Z}= & \partial^{\mu} Z_{\mu}+\xi_{z}\left(M_{Z} \chi_{3}+\frac{e}{2 s_{W} c_{W}} \tilde{\varepsilon} H \chi_{3}\right) \\
F^{A}= & \partial^{\mu} A_{\mu} .
\end{aligned}
$$

Here, $\tilde{\alpha}, \tilde{\beta}, \tilde{\delta}$, $\tilde{\varepsilon}$, and $\tilde{\kappa}$ are nonlinear gauge parameters specific to this formalism. The parameters $s_{W}$ and $c_{W}$ are the sine and cosine of Weinberg angle $\theta_{W}$. In our calculation we set $\xi=\xi_{W}=\xi_{Z}=1$ to make the gauge boson propagators simple. NLG parameters are distributed among so many diagrams of different types of topologies. So it is very powerful if we can verify the cancellation of these NLG parameters. We show the sample of cancellation in Appendix F.

\section{Successive method}

Diagrams with self-energy type two-point function can be calculated by successive method using renormalized two point function. An example is diagram with $(\gamma-\gamma)$ or $(\gamma-Z)$ vacuum polarization type diagrams. We decompose the renormalization constants $\delta Z_{A A}^{1 / 2}, \delta Z_{Z A}^{1 / 2}, \delta Z_{A Z}^{1 / 2}, \delta M_{Z}^{2}$ etc. into components according to the particles involved in the loop. By adding the counterterm to corresponding one-loop unrenormalized two-point function, one-loop $\left(\ell_{1}\right)$ integration is performed without divergence and we obtain the renormalized two-point function $\Pi_{R}$. By inserting $\Pi_{R}$ into the second $\operatorname{loop}\left(\ell_{2}\right)$, we get finite value of $a_{\mu}$. We use this alternative method to reconfirm the results obtained by the methods given in Sec. III. An example is shown in Appendix G.

\section{RESULTS OF OUR CALCULATION}

As the physical input parameters, we use the following fermion and boson masses (unit $\mathrm{GeV}$ ). $m_{\mu}=$ $105.6583745 \times 10^{-3}, m_{e}=0.5109989461 \times 10^{-3}, m_{\tau}=$ $1.77686 m_{u}=0.3, \quad m_{c}=1.5, \quad m_{t}=173.1, \quad m_{d}=0.3$, $m_{s}=0.5, \quad m_{b}=4.18, \quad M_{W}=80.385, \quad M_{Z}=91.1876$, $M_{H}=125.09$. We also choose the fine structure constant in the Thomson limit, $\alpha=1 / 137.035999139$.

After clearing all the conditions given in Sec. IV B we

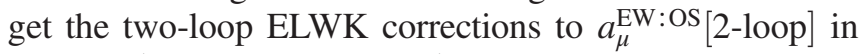
terms of $\left(\alpha, M_{Z}, M_{W}, M_{H}, m_{f}\right)$. The loop expansion is done by using $\alpha, \alpha^{2}$, successively. Among 1780 diagrams, we exclude 9 pure QED diagrams consisting of only $(e, \mu, \tau, \gamma)$ and 6 diagrams containing vacuum polarization composed of quark loop. Then the final result becomes,

$$
a_{\mu}^{\mathrm{EW}: O S}[2-\text { loop }]=(-36.76 \pm 0.3) \times 10^{-11} .
$$


The errors in the above and the following expressions are limited to the numerical integration error and the uncertainty of input parameters $M_{W}, M_{H}, M_{Z}, m_{t}, m_{b}$. The masses of light quarks are fixed in our model.

We show the fermionic and bosonic part of two loop correction separately for reference.

$$
\begin{aligned}
& a_{\mu}^{\mathrm{EW}: \mathrm{OS}}[2 \text {-loop }]_{\text {fermion }}=-18.34( \pm 0.2), \\
& a_{\mu}^{\mathrm{EW}: \mathrm{OS}}[2-\text { loop }]_{\mathrm{boson}}=-18.42( \pm 0.1) .
\end{aligned}
$$

As we mentioned before, we adopt OS renormalization, however, the expression in the preceding works is parametrized using Fermi constant $G_{F}=1.1663787 \times$ $10^{-5} \mathrm{GeV}^{-2}$ and $\alpha$ [17].

The difference of the 2-loop correction between our value and that in $G_{F}$ parametrization is due to the fact that one loop correction in $G_{F}$ parametrization partially includes the $\alpha^{2}$ correction in our scheme.

So the comparison should be done to the sum of one- and two-loop. The one loop correction in our OS scheme is written down as follows.

$$
\begin{aligned}
a_{\mu}^{\mathrm{EW}: \mathrm{OS}}[1-\text { loop }]= & \frac{\alpha m_{\mu}^{2} M_{Z}^{2}}{16 \pi\left(M_{Z}^{2}-M_{W}^{2}\right) M_{W}^{2}} \\
& \times\left[\frac{5}{3}+\frac{1}{3}\left(\frac{4 M_{W}^{2}}{M_{Z}^{2}}-3\right)^{2}+\mathcal{O}\left(\frac{m_{\mu}^{2}}{M_{W}^{2}}\right)\right. \\
& \left.+\mathcal{O}\left(\frac{m_{\mu}^{2}}{M_{H}^{2}}\right)\right] .
\end{aligned}
$$

We can carry out the numerical calculation without any approximation and get the value.

$$
a_{\mu}^{\mathrm{EW}: \mathrm{OS}}[1 \text {-loop }]=187.99( \pm 0.2) \times 10^{-11} .
$$

By summing up one and two loop weak corrections, our result is as follows.

$$
a_{\mu}^{\mathrm{EW}: \mathrm{OS}}[1+2 \text {-loop }]=151.2( \pm 1.0) \times 10^{-11} .
$$

Here we add the error due to neglecting the uncertainty in electroweak loops involving hadrons.

When we compare our result with the value obtained by using $G_{F}$ parametrization, we need the naive free light quark model calculation with the same quark masses as ours. This is given ${ }^{3}$ in Ref. [15]. In this case the two loop correction becomes, $-42.97( \pm 1) \times 10^{-11}$. In the $G_{F}$ parametrization, the one loop correction becomes $194.80( \pm 0.01) \times 10^{-11}[17]$, so that we get,

$$
a_{\mu}^{\mathrm{EW}}[1+2-\text { loop }]_{\mathrm{FQM}}=151.8( \pm 1) \times 10^{-11} .
$$

This is consistent with our value Eq. (22).

\footnotetext{
${ }^{3}$ In Ref. [15], the contribution of light quarks in $\mathrm{FQM}$ is $a_{\mu}^{\mathrm{EW}(2)}(e, \mu, u, c, d, s)=-(4.0+4.65) \times 10^{-11}=-8.65 \times 10^{-11}$.
}

We also add a comment on the relation between the wellknown PDG value [20] shown below and our value. If we include the hadronic correction to light quark contribution, by adding the difference of the following expression [17],

$a_{\mu}^{\mathrm{EW}(2)}(e, \mu, u, c, d, s)=(-6.91 \pm 0.20 \pm 0.3) \times 10^{-11}$

and the value quoted in the footnote below [15], our value becomes as follows.

$$
a_{\mu}^{\mathrm{EW}}[1+2 \text {-loop }]=152.9( \pm 1) \times 10^{-11} .
$$

It is in agreement with the following PDG value [20] within errors.

$$
a_{\mu}^{\mathrm{EW}}=153.6( \pm 1) \times 10^{-11} .
$$

\section{DISCUSSIONS AND COMMENTS}

We developed the system to calculate the full ELWK twoloop corrections to $a_{\mu}$, by fully using GRACE and FORM on the basis of OS-scheme. The work we need beforehand is only to prepare several files which only depend on the type of the topology of diagrams as we explained in Sec. III. We adopt the dimensional regularization to regularize UV- and IR-divergences and to get finite gauge invariant values of the physical quantity. To extract the $(1 / \varepsilon)$ terms, we use the linear extrapolation method explained in Sec. III B. This method is very simple and attractive, compared with the conventional method to take out the $(1 / \varepsilon)$ term by extrapolating one of the Feynman parameters close to 0 . If we adopt the conventional method, it is crucial to introduce the most suitable transformations from $\left(z_{j}\right)$ to $[0,1]$ integration variables $(x, y, u, v, w)$. Furthermore, we need rather complex operations including differentiation of the amplitude, etc. As a result, the necessary CPU-time increases extensively.

In the case of the linear extrapolation method (LE), however, the choice of integration variables is not sensitive to get the reliable results and this method decreases the number of operation drastically. It is sufficient to define the quantity as a function of $\varepsilon(=2-n / 2)$. We only need to treat Dirac matrices and various vectors appeared in the numerator, in $n$-dimension. This is easily done by using symbolic manipulation system such as FORM. The operation is simple and we can make use of the resultant short sources for both $\mathrm{UV}-(\varepsilon>0)$ and IR- $\left(\varepsilon_{R}=-\varepsilon>0\right)$ regularization and also to get finite results. We conclude that LE-method is the most simple and reliable method, at this moment. In order to get reliable physical value by this method, high precision numerical integration over Feynman parameters is inevitable. The DE-method introduced in Sec. IVA is the suitable candidate.

Introduction of NLG-parameters makes the calculation very complex, however, it is very powerful to check the calculation of so called Boson contribution. The number of diagrams consisting of different types of topology are 
connected through NLG-parameters. The maximum number of diagrams mutually entangled reaches 864 . So this is a very tough condition to be cleared.

By making use of these technical approaches mentioned above, we clear all the constraints given in Sec. IV B. Namely, (i) reproduction of QED values, (ii)(iii) cancellation of UV-and IR-divergences, (iv) independence of NLG-gauge parameters. We show some samples in the Appendices how they are cleared.

The final value of the sum of one and two loop weak corrections is approximately the same as the one obtained by previous works using different parametrization.

Based on this work we can proceed to construct PNQFT (perturbative numerical quantum field theory), which we discussed in Sec. I A. Wide range of application to ELWK higher loop expansion for several physical reactions will be opened. We expect that this work provides the fruitful foundation to formulate PNQFT.

\section{ACKNOWLEDGMENTS}

We would like to thank Prof. T. Kaneko for his important contribution to construct the framework of calculation at the early stage of this work. We also wish to thank Prof. K. Kato, Prof. F. Yuasa, and Prof. M. Kuroda for discussions. Last but not least, we express our deep appreciation to the late Prof. Y. Shimizu for his continual encouragement. This research is partially supported by Grant-in-Aid for Scientific Research $(15 \mathrm{H} 03668,15 \mathrm{H} 03602)$ of JSPS and Grand-in-Aid for High Performance Computing with General Purpose Computers (Research and development in the next-generation area) of MEXT.

\section{APPENDIX A: REPRODUCTION OF QED TWO-LOOP VALUE}

QED two-loop value is reproduced correctly.

\begin{tabular}{lc}
\hline & Unit $=(\alpha / \pi)^{2}$ \\
\hline Analytic expression & -0.328478996 \\
Our value & -0.328479821 \\
\hline
\end{tabular}

\section{APPENDIX B: CLASSIFICATION OF DIAGRAMS TO CHECK NUMERICAL VALUES}

In Fig. 1, we show types of topology to formulate the two-loop contributions. However, in order to check the
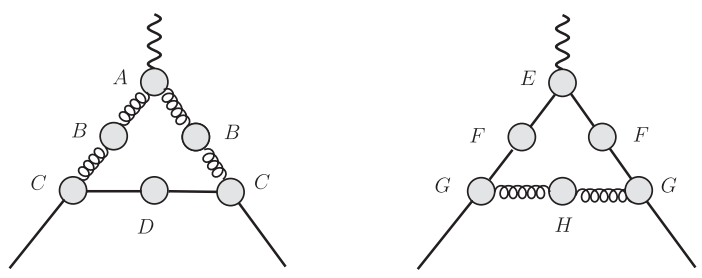

FIG. 2. Classification of diagrams.
TABLE I. Classification of two loop diagrams. Depending on the position $(\mathrm{A} \sim \mathrm{H})$ of one loop diagram or one loop counterterm, we give names shown below. For example, LAD-I and LAD-II correspond to topology (3) in Fig. 1. The typical diagram belonging to LAD-I is fermion triangle with $\gamma-\gamma-Z$ legs.

\begin{tabular}{|c|c|c|c|}
\hline $\mathrm{A} \rightarrow$ LAD-I & $\mathrm{B} \rightarrow$ SLF-I & $\mathrm{C} \rightarrow \mathrm{VTX}-1$ & $\mathrm{D} \rightarrow \mathrm{VCP}-\mathrm{I}$ \\
\hline $\mathrm{E} \rightarrow \mathrm{LAD}-\mathrm{II}$ & $\mathrm{F} \rightarrow \mathrm{SLF}-\mathrm{II}$ & $\mathrm{G} \rightarrow \mathrm{VTX}-\mathrm{II}$ & $\mathrm{H} \rightarrow$ VCP-II \\
\hline \multicolumn{2}{|l|}{ Fig. 1(1) } & \multicolumn{2}{|c|}{ Fig. 1(6) $\rightarrow$ DBT } \\
\hline
\end{tabular}

consistency of numerical values, it is useful to classify diagrams by distinguishing fermion and boson lines in each diagrams in Fig. 1. We briefly figure out the classification method in Fig. 2.

In the figure, the straight lines and wavy lines represent fermion and boson, respectively. The circle indicates the one-loop diagram. We classify diagrams depending on the place where the one-loop diagram is inserted. It is summarized in the following Table I. You can easily see which one of Fig. 1 is classified into which category.

In the above Table I we add two types of topology having no divergence, namely, Fig. 1-(1) and (6).

\section{APPENDIX C: UV-CANCELLATION}

In this Appendix, we show the cancellation of UVdivergence in linear gauge ('t Hooft-Feynman gauge). Examples of a group of diagrams are shown in Fig. 3. The diagrams in Fig. 3 belong to several groups in Table I.

In this case, total 13 diagrams and 1 counterterm (e) make a group to cancel UV-divergence. In the Table II, the coefficient of $C_{\mathrm{UV}}^{(2)}=(1 / \varepsilon-2 \gamma+2 \ln (4 \pi))$, corresponding to each diagram is shown. As you can read from the Table II, the cancellation is marvelous, up to almost 15 digits.

\section{APPENDIX D: IR-CANCELLATION}

LE method is applied to check IR-cancellation. Among two-loop diagrams, the 8 diagrams in Fig. 4 have IRdivergence. The diagrams with $\mathrm{CT}$ also have IR-divergence

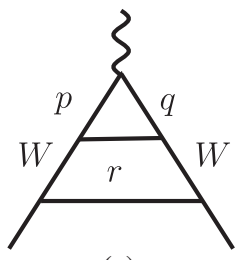

(a)

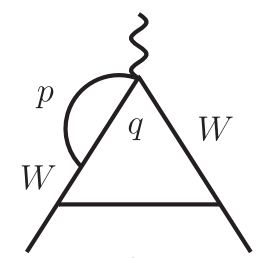

(b)

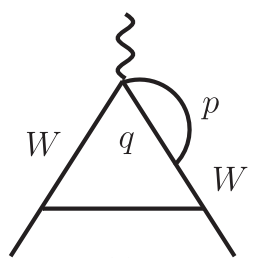

(c)

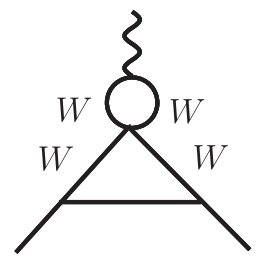

(d)

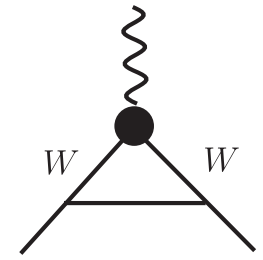

(e)
FIG. 3. Sample diagrams with $W$. 
TABLE II. Sample of UV cancellation in 't Hooft-Feynman gauge. UV cancellation is confirmed by summing up the coefficient of $C_{\mathrm{UV}}^{(2)}$ of a set of diagrams. Here we show the set given in Fig. 3 as a sample. The 15-digit cancellation is realized in the sum.

\begin{tabular}{lcc}
\hline \hline $\begin{array}{l}\text { Diagram in } \\
\text { Fig. 3 }\end{array}$ & $\begin{array}{c}\text { Particles on line } \\
(p, q, r)\end{array}$ & Value (unit $\left.10^{-11}\right)$ \\
\hline (a) & $W-W-Z$ & 1.72758038865755734 \\
(a) & $W-W-\gamma$ & 0.49552373441415571 \\
(a) & $\chi-\chi-H$ & 0.05700266982235161 \\
(a) & $\chi-\chi-\chi 3$ & 0.05700266982235161 \\
(a) & $c^{-}-c^{-}-c^{Z}$ & -0.02214846652125073 \\
(a) & $c^{-}-c^{-}-c^{\gamma}$ & -0.00635286838992507 \\
(a) & $c^{+}-c^{+}-c^{Z}$ & -0.02214846652125073 \\
(a) & $c^{+}-c^{+}-c^{\gamma}$ & -0.00635286838992507 \\
(b) & $W-Z$ & -0.17085951453900741 \\
(b) & $W-\gamma$ & -0.04900781767402955 \\
(c) & $W-Z$ & -0.17085951453900741 \\
(c) & $W-\gamma$ & -0.04900781767402955 \\
(d) & & -2.63840950598091337 \\
(e) & Sum & -0.00000000000000012 \\
\hline \hline
\end{tabular}

through $\delta Z_{W}^{1 / 2}$ (19 diagrams) and $\delta Z_{\mu}^{1 / 2}$ (28 diagrams). The IR-divergence at two-loop level is proportional to $C_{\mathrm{IR}}^{(2)}=\left(-1 / \varepsilon_{R}-2 \gamma+2 \ln (4 \pi)\right), \varepsilon_{R}=(n / 2-2)>0$. It is easily shown that the IR-divergence coming from $\delta Z_{W}^{1 / 2}$ cancels among the 19 CT-diagrams. As for the diagrams with $\delta Z_{\mu}^{1 / 2}$, IR-divergence is cancelled by the corresponding two-loop diagrams. We show coefficients of $C_{\mathrm{IR}}^{(2)}$ in Table III. The correspondence between small photon mass $(\lambda)$ method and LE-method for IR-regularization is checked in the case of QED ladder diagram. Analytic value of a coefficient of $\ln \left(\lambda^{2} / m_{\mu}^{2}\right)$ in unit of $(\alpha / \pi)^{2}$ is (1/4) [9]. It is

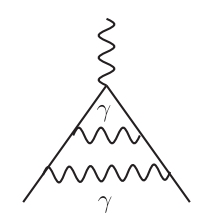

(a)

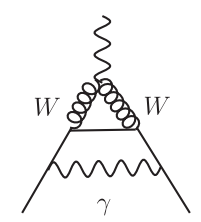

(e)

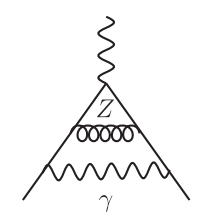

(b)

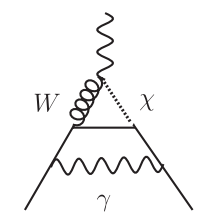

(f)

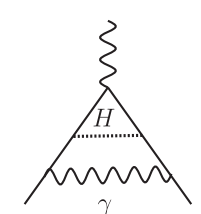

(c)

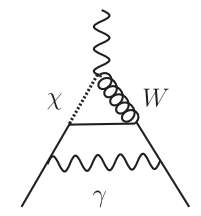

(g)

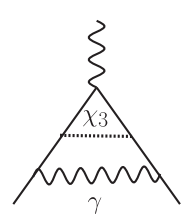

(d)

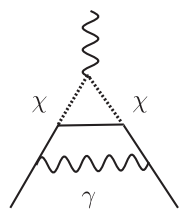

(h)
FIG. 4. All the two-loop diagrams containing IR-divergence.
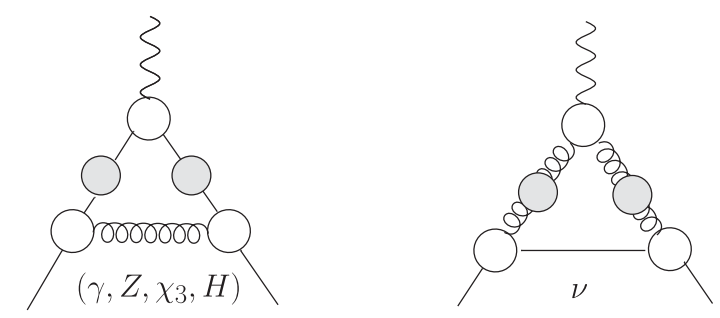

FIG. 5. Diagrams containing IR-divergence through $\delta Z$. The straight lines and wavy lines represent fermion and boson, respectively. The circle represent $\delta Z_{\mu}^{1 / 2}$ or $\delta Z_{W}^{1 / 2}$. In the case where the corner includes $\nu$-particle, both of $\delta Z_{\mu}^{1 / 2}, \delta Z_{W}^{1 / 2}$ are taken into account. The gray circles and white circles correspond to self CT and vertex CT in Table III, respectively.

0.249999998 by our calculation using small photon mass. In the LE method, the coefficient of $C_{\mathrm{IR}}^{(2)}$ becomes 0.249999999 . We understand the correspondence between $\ln \left(\lambda^{2} / m_{\mu}^{2}\right)$ and $C_{\mathrm{IR}}^{(2)}$ is established. As we show in Table III, no IR-divergence remains in the final expression.

\section{APPENDIX E: SAMPLE CALCULATION OF THE TWO-LOOP DIAGRAM}

As a sample, we show the calculation of the two-loop diagram which contains both UV- and IR-divergences. The diagram is shown in Fig. 4(b). The line numbers are given in Fig. 6. Following Eq. (9) given in Sec. III A, the essential part of expression of two-loop diagram contribution is written as the following form.

$$
\begin{gathered}
F_{0}=\int \prod d z_{j}\left[\frac{1}{(\operatorname{det} \mathrm{U})^{-3 \varepsilon}} \frac{f_{0}}{(\mathscr{D}-i \epsilon)^{2+2 \varepsilon}}\right] \\
F_{2}=\int \prod d z_{j}\left[\left(-\frac{1}{2}\right) \frac{1}{(\operatorname{det} \mathrm{U})^{2-3 \varepsilon}} \frac{f_{2}}{(\mathscr{D}-i \epsilon)^{1+2 \varepsilon}}\right] .
\end{gathered}
$$

Quantities in Eqs. (E1), (E2) are expressed by Feynman parameters $z_{j},(j=1, \ldots, 6),\left(\sum_{j} z_{j}=1\right)$. Masses are made dimensionless using muon mass.

$$
\begin{aligned}
V= & -z_{5}+z_{5}^{2}\left(z_{23}+z_{6}\right) / \operatorname{det} \mathrm{U}+z_{1234}+z_{6} M_{Z}^{2}, \\
\operatorname{det} \mathrm{U}= & z_{6} z_{12345}+z_{3} z_{45}+z_{2} z_{145}+z_{1} z_{3}, \\
\mathscr{D} \equiv & \operatorname{det} \mathrm{U} \times V=\left(z_{14}+z_{23}\right) z_{14} z_{23}+z_{23}^{2} z_{5} \\
& +\left(z_{14}+z_{23}\right)^{2} z_{6}+\left(z_{6} M_{Z}^{2}\right) \operatorname{det} \mathrm{U}
\end{aligned}
$$

where $z_{i j \cdots k}=z_{i}+z_{j}+\cdots+z_{k}$. Functions $f_{0}, f_{2}$ are expanded in $\varepsilon$ up to $O(\varepsilon)$.

$f_{0}=f_{00}+\varepsilon f_{01}, \quad f_{2}=f_{20}+\varepsilon f_{21}, \quad \varepsilon=(2-n / 2)$.

The $f_{0}$ and $f_{2}$ part give IR- and UV-divergences, respectively. By adopting DE and LE methods for Feynman 
TABLE III. Cancellation of IR-divergence is shown. IR cancellation is confirmed by summing up the coefficient of $C_{\mathrm{IR}}^{(2)}$. All the diagrams having IR-divergence are shown in Fig. 4. Typical diagrams having IR-divergence through the renormalization constants are shown in Fig. 5.

\begin{tabular}{|c|c|c|c|c|}
\hline Type of correction & Numerical Value (unit $10^{-11}$ ) & & & \\
\hline Diagram Type & $\delta Z_{\mu}^{1 / 2}$ Vertex CT & $\delta Z_{\mu}^{1 / 2}$ Self CT & Two-loop diagram & Sum \\
\hline Neutral Type & & & Diagram & \\
\hline$\gamma$ & $-134887.2756 \times 3$ & $134887.2756 \times 2$ & (a) +134887.2755 & $-1.6 \times 10^{-4}$ \\
\hline$Z-$ boson & $0.217294 \times 3$ & $-0.217294 \times 2$ & (b) -0.217294 & $-2.0 \times 10^{-9}$ \\
\hline Higgs, $\chi_{3}$ & $1.67970 \times 10^{-6} \times 3$ & $-1.67970 \times 10^{-6} \times 2$ & $(c, d)-1.67970 \times 10^{-6}$ & $2.6 \times 10^{-13}$ \\
\hline \multicolumn{5}{|l|}{ Charged type } \\
\hline$W^{ \pm}, \chi^{ \pm}$ & -0.435625 & ---- & (e)-(h) 0.435625 & $+3.4 \times 10^{-12}$ \\
\hline
\end{tabular}

parameter integration, we get the following expansion. $\left(\varepsilon_{R}=-\varepsilon\right)$

$$
\begin{aligned}
& F_{0} \rightarrow\left(\frac{C_{-1}}{-\varepsilon_{R}}+C_{0}+C_{1}\left(-\varepsilon_{R}\right)+\cdots\right), \\
& F_{2} \rightarrow\left(\frac{D_{-1}}{\varepsilon}+D_{0}+D_{1} \varepsilon+\cdots\right) .
\end{aligned}
$$

We multiply the $\Gamma$ functions arising from n-dimensional integration and factor $1 /(4 \pi)^{n}$, to the above quantities,

$$
\begin{aligned}
& \frac{\Gamma(6-n)}{(4 \pi)^{n}} F_{0} \rightarrow\left[C_{-1} C_{\mathrm{IR}}^{(2)}+2 C_{-1}+C_{0}+O\left(\varepsilon_{R}\right)\right], \\
& \frac{\Gamma(5-n)}{(4 \pi)^{n}} F_{2} \rightarrow\left[D_{-1} C_{\mathrm{UV}}^{(2)}+D_{0}+O(\varepsilon)\right] .
\end{aligned}
$$

We must pay attention that the term $\left(2 C_{-1}\right)$ appears, because $\Gamma(6-n)$ contains the term $\left(1-2 \varepsilon_{R}\right)$. In order to get the correct finite value, we must calculate the counterterms using the same regularization method for both IRand UV-divergences as in two-loop case. In this case, we need muon wave function renormalization constant $\delta Z_{\mu}$. The $\delta Z_{\mu}$ is obtained by calculating muon self-energy diagrams. The $\delta Z_{\mu}(\gamma)$ represents photon exchange part and $\delta Z_{\mu}(Z)$ represents $Z$-exchange part.

In Fig. 6(b), $C T_{Z}$ represents $\delta Z_{\mu}(Z)$, and in Fig. 6(c), $C T_{1} \sim C T_{5}$ represent only the IR-divergent part of $\delta Z_{\mu}(\gamma)$. These IR-divergent part cancels that of Fig. 6(a) as is shown

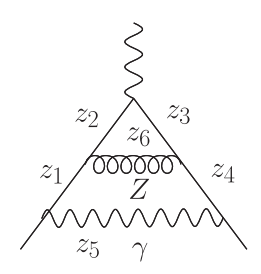

(a)

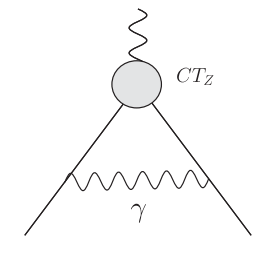

(b)

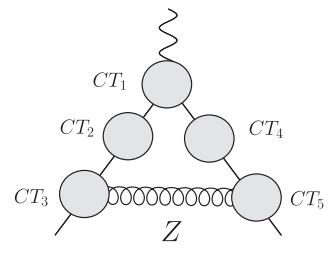

(c)
FIG. 6. Diagram containing both UV- and IR-divergences. $C T_{Z}, C T_{1} \sim C T_{5}$ represent the CT-terms. in the Table III. We will show the numerical results to see the situation clearly. (unit $=10^{-11}$ )

$$
\begin{aligned}
C_{-1} & =-0.217294, \\
C_{0} & =1.672727 \\
D_{-1} & =24621.375584, \\
D_{0} & =-246732.662539 .
\end{aligned}
$$

The term $C T_{Z}$ has both UV and finite parts.

$$
\begin{aligned}
\left(C T_{Z}\right)_{\mathrm{UV}} & =-24621.375584, \\
\left(C T_{Z}\right)_{f} & =246728.863173 .
\end{aligned}
$$

UV-cancellation is excellent and cancels 17 digits.

$$
D_{-1}+\left(C T_{Z}\right)_{\mathrm{UV}}=3.84 \times 10^{-12} .
$$

To cancel IR part of Fig. 6(a), we adopt IR-part of $\delta Z_{\mu}(\gamma)$, $C T_{1} \sim C T_{5}$. It is explained in the Table III, row ( $Z$ boson). The finite value is obtained as follows.

$$
C_{0}+2 C_{-1}+D_{0}+\left(C T_{Z}\right)_{f}=-2.561228 .
$$

We can also regularize the IR divergent part by employing the small photon mass $\lambda^{2}$, to see $\log \left(\lambda^{2}\right)$ term. As we notice that we must use the same regularization method for both two-loop and CT diagrams. At first sight, the finite part changes compared with the dimensional regularization method, however, the sum of two-loop and CT contribution is the same in both regularization methods. We confirm it by numerical calculation. As we can see from Eq. (E9), we need very careful treatment to discuss order of (a few) $\times 10^{-11}$ quantity of $a_{\mu}$.

\section{APPENDIX F: NLG PARAMETER CANCELLATION}

As an example of cancellation of NLG parameters, we show the UV-divergent part and finite part of diagrams which contain $\tilde{\alpha}^{n}(n=1,2,3)$ terms. Total number of 
TABLE IV. Cancellation of NLG $\tilde{\alpha}$ parameter is shown. Numbers are in unit of $10^{-11}$. All the two-loop diagrams and CT terms are classified according to the Table II. Numbers represent the coefficients of $\tilde{\alpha}, \tilde{\alpha}^{2}, \tilde{\alpha}^{3}$ after summing up the contributions of both two-loop diagrams and CT terms. The last row shows the maximum absolute value within the same column and its topology type, to show how the cancellation works well.

\begin{tabular}{|c|c|c|c|c|c|c|c|c|}
\hline \multirow[b]{2}{*}{ Type of Diagrams } & \multicolumn{2}{|c|}{ Number of Diagrams } & \multicolumn{3}{|c|}{ Coefficient of $C_{\mathrm{UV}}^{(2)}$ (unit $10^{-11}$ ) } & \multicolumn{3}{|c|}{ Finite contribution to (g-2) (unit $10^{-11}$ ) } \\
\hline & 2loop & $\mathrm{CT}$ & $\tilde{\alpha}$ & $\tilde{\alpha}^{2}$ & $\tilde{\alpha}^{3}$ & $\tilde{\alpha}$ & $\tilde{\alpha}^{2}$ & $\tilde{\alpha}^{3}$ \\
\hline LAD-I & 400 & 10 & $1.3 \times 10^{-11}$ & -0.25876 & -0.03235 & 6.11086 & 3.98570 & 0.83255 \\
\hline LAD-II & 468 & 6 & $-1.0 \times 10^{-11}$ & & & -0.12093 & & \\
\hline VTX-I & 80 & 8 & $1.1 \times 10^{-16}$ & $-5.7 \times 10^{-16}$ & & 1.16937 & 2.07396 & \\
\hline VTX-II & 90 & 8 & $-4.7 \times 10^{-11}$ & & & -0.83645 & & \\
\hline SLF-I & 312 & 16 & $3.6 \times 10^{-16}$ & 0.25876 & 0.03235 & -3.48758 & -3.87681 & -0.83255 \\
\hline SLF-II & 48 & 8 & & & & & & \\
\hline VCP-I & 12 & 4 & $-1.1 \times 10^{-10}$ & $-1.1 \times 10^{-10}$ & & & & \\
\hline VCP-II & 280 & 10 & & & & -0.05656 & 0.00074 & \\
\hline CRL & 72 & 0 & & & & -2.77731 & 1.18564 & \\
\hline DBT & 18 & 0 & & & & & -3.36911 & \\
\hline SUM & 1780 & 70 & $-1.65 \times 10^{-10}$ & $-1.42 \times 10^{-10}$ & $<1 \times 10^{-30}$ & 0.00138 & 0.00012 & $1.1 \times 10^{-12}$ \\
\hline Type of diagram & & & VTX-II & LAD-I & SLF-I & VTX-I & LAD-I & SLF-I \\
\hline Max. absolute value & & & -298325.54724 & -8.32905 & -0.19407 & 2763874.32283 & -46.38364 & 4.41044 \\
\hline
\end{tabular}

${ }^{\mathrm{a}}$ Each contribution is calculated in quadruple precision method and has more effective digits than shown in the table.

diagrams depending on $\tilde{\alpha}$ amount to 864. In order to see how the NLG-parameters are cancelled, we classify diagrams according to Fig. 2 and Table I. We summarize the result of numerical calculation in Table IV. In the last row in Table IV, we show the maximum absolute value among the individual terms in the column and its type of diagram, to indicate the degree of the cancellation. First we see the cancellation of the parameter $\tilde{\alpha}^{n}$ in the UV-divergent part, namely, the coefficients of $C_{\mathrm{UV}}^{(2)}$. It is shown in the left half part of the Table IV. We can see the cancellation works very well and it strongly guarantees the validity of our numerical calculation. In the right half of Table IV, we also show the finite contribution to $a_{\mu}$. The column $\tilde{\alpha}^{1} \sim \tilde{\alpha}^{3}$ show that how the NLG-cancellation works well also in finite part.

\section{APPENDIX G: SUCCESSIVE METHOD}

In some cases, in order to check our calculation, we perform two-loop integrations successively. By integrating the first loop $\left(\ell_{1}\right)$, we make the renormalized effective function and insert it to the second loop $\left(\ell_{2}\right)$ integration. As an example, we calculate the diagrams consisting of photon- $\mathrm{Z}$ meson mixing vacuum polarization. There are two types of topology 4 and 10 in Fig. 1.

In general, unrenormalized two point $\gamma-Z$ function is written as follows.

$\Pi\left(q^{2}\right)_{\mu \nu}^{u n}=\Pi_{T}^{u n}\left(q^{2}\right)\left(g_{\mu \nu}-\frac{q_{\mu} q_{\nu}}{q^{2}}\right)+\Pi_{L}^{u n}\left(q^{2}\right) \frac{q_{\mu} q_{\nu}}{q^{2}}$.

Each one-loop diagram contributes to $\Pi_{T}^{u n}\left(q^{2}\right), \Pi_{L}^{u n}\left(q^{2}\right)$ in the following form.
$\Pi_{T}^{u n}\left(q^{2}\right)=a\left(q^{2}\right), \quad \Pi_{L}^{u n}\left(q^{2}\right)=a\left(q^{2}\right)+q^{2} b\left(q^{2}\right)$

where

$a\left(q^{2}\right)=\int \frac{d^{n} \ell}{i(2 \pi)^{n}}\left[\frac{A_{1} \ell^{2}+\left(A_{2} q^{2}+A_{3}\right)}{\left(\ell^{2}-D_{Q}\right)^{2}}+\frac{A_{4}}{\left(\ell^{2}-m^{2}\right)}\right]$,

$b\left(q^{2}\right)=\int \frac{d^{n} \ell}{i(2 \pi)^{n}} \frac{B}{\left(\ell^{2}-D_{Q}\right)^{2}}$.

The variables $A_{1} \sim A_{4}, B, D_{Q}, m$ depend on the specific one-loop diagram. The $A_{4}$-term comes from the 4-point boson coupling diagram. However, this term drops out by the renormalization process.

In the followings, the renormalized quantity $\Pi$ are expressed as $\hat{\Pi}$.

$$
\begin{aligned}
\hat{\Pi}_{T}\left(q^{2}\right) & =\left(M_{Z}^{2}-q^{2}\right) \delta Z_{Z A}^{1 / 2}-q^{2} \delta Z_{A Z}^{1 / 2}+\Pi_{T}^{u n}\left(q^{2}\right), \\
\hat{\Pi}_{L}\left(q^{2}\right) & =M_{Z}^{2} \delta Z_{Z A}^{1 / 2}+\Pi_{L}^{u n}\left(q^{2}\right) \\
\hat{\Pi}_{T}\left(q^{2}=0\right) & =0, \quad \hat{\Pi}_{T}\left(q^{2}=M_{Z}^{2}\right)=0
\end{aligned}
$$

We notice that the renormalization conditions are fixed by $\Pi_{T}^{u n}\left(q^{2}\right)$, there is no freedom to renormalize $\Pi_{L}^{u n}\left(q^{2}\right)$. By using these renormalization conditions, we can write down renormalized $a_{R}\left(q^{2}\right)$. It has no divergence, we can perform loop $\left(\ell_{1}\right)$ integration. The result is given as Eq. (G5),(G6). where $M$ represents the mass of a particle circulating the loop. As for $\hat{b}\left(q^{2}\right)$, we must check whether it is really finite or not. The $C_{\mathrm{UV}}$-part of $\hat{b}\left(q^{2}\right)$ disappears after summing all the one-loop diagrams and integration of Feynman parameter $x$. 


$$
\begin{aligned}
\hat{a}\left(q^{2}\right)= & \hat{\Pi}_{T}^{A Z}\left(q^{2}\right)=\left(\frac{1}{16 \pi^{2}}\right) \int_{0}^{1} d x \int_{0}^{1} d z\left[A_{1}\left\{q^{2}\left(q^{2}-M_{Z}^{2}\right)\right\}\left[x(1-x)^{2}\right] \frac{-2}{-z x(1-x)} \frac{1}{\left(q^{2}-M_{3}^{2}\right)}\right. \\
& +A_{2}\left\{q^{2}\left(q^{2}-M_{Z}^{2}\right)\right\}[x(1-x)] \frac{1}{-z x(1-x)} \frac{1}{\left(q^{2}-M_{2}^{2}\right)}+A_{3}\left\{q^{2}\right\}[x(1-x)] \frac{1}{-z x(1-x)} \frac{1}{\left(q^{2}-M_{1}^{2}\right)} \\
& \left.+A_{3}\left\{q^{2}\right\}[x(1-x)] \frac{-1}{V_{W Z}}\right],
\end{aligned}
$$

$$
\begin{aligned}
M_{1}^{2} & =\frac{M^{2}}{z x(1-x)}, \\
M_{2}^{2} & =\frac{M^{2}}{z x(1-x)}-\frac{1-z}{z} M_{Z}^{2}, M_{3}^{2}=\frac{M^{2}}{z x(1-x)}-\frac{y}{z} M_{Z}^{2}, \\
V_{W Z} & =M^{2}-z x(1-x) M_{Z}^{2} .
\end{aligned}
$$

We also notice that the $\hat{b}\left(q^{2}\right)$-part does not contribute to $a_{\mu}$, because it is proportional to $q_{\mu} q_{\nu}$.

The next step is to insert the renormalized two point function $\hat{a}\left(q^{2}\right) g_{\mu \nu}$ into the triangle diagram and integrate over second loop momentum $\ell_{2}$. The integration has logarithmic divergent part, however, it drops out by the projection operator to $a_{\mu}$, Eq. (8). Final expression is very complex and we do not quote here. From Eq. (8), we can see that there are denominators having $\ell_{2}(\leftarrow q)$. So the final expression has 5 integration parameters which run in the interval $[0,1]$. As a sample of calculation, we show sum of boson loop contribution. Fish type diagrams, $\left\{(W W),(W \chi),(\chi W),(\chi \chi),\left(c^{+} c^{+}\right),\left(c^{-} c^{-}\right)\right\}$and tad pole type diagrams, $\left\{(W),(\chi),\left(c^{+}\right),\left(c^{-}\right)\right\}$composing $\Pi\left(q^{2}\right)_{\mu \nu}$. In unit of $10^{-11}$ we get, $-6.1048 \times 10^{-3}$ by this method. On the other hand, we get $-6.104 \times 10^{-3}$ by our two-loop formalism with CT-terms. The coincidence is quite good. Notice that, in this case the two-loop formalism takes huge cpu-time, especially for $(W-W)$ diagram. Its contribution is around $8.8 \times 10^{6}$ in unit of $10^{-11}$ so that we need almost a 15 digit number to cancel UV-divergence. So, the effective method is not only important to check the reliability of our general formalism but also is useful to get the numerical result. In the case of self-energy type diagrams, we can construct effective method in several cases, however, for vertex type diagrams, to construct effective method is complex.
[1] F. Yuasa et al., Prog. Theor. Phys. Suppl. 138, 18 (2000).

[2] T. Aoyama, M. Hayakawa, T. Kinoshita, and M. Nio, Phys. Rev. Lett. 109, 111807 (2012).

[3] T. V. Kukhto, E. A. Kuraev, Z. K. Silagadze, and A. Schiller, Nucl. Phys. B371, 567 (1992).

[4] R. Jackiw and S. Weinberg, Phys. Rev. D 5, 2396 (1972).

[5] G. Altarelli, N. Cabibbo, and L. Maiani, Phys. Lett. 40B, 415 (1972).

[6] I. Bars and M. Yoshimura, Phys. Rev. D 6, 374 (1972).

[7] K. Fujikawa, B. W. Lee, and A. I. Sanda, Phys. Rev. D 6, 2923 (1972).

[8] T. Kaneko and N. Nakazawa, Artificial intelligence in highenergy and nuclear physics '95, in Proceedings of the 4th International Workshop On Software Engineering, Artificial Intelligence, and Expert Systems, Pisa, Italy, 1995 (World Scientific, Singapore, 1995), pp. 0173-178.

[9] R. Karplus and N. M. Kroll, Phys. Rev. 77, 536 (1950).

[10] C. M. Sommerfield, Phys. Rev. 107, 328 (1957).

[11] A. Petermann, Helv. Phys. Acta 30, 407 (1957).

[12] C. M. Sommerfield, Ann. Phys. (N.Y.) 5, 26 (1958).

[13] A. Czarnecki, B. Krause, and W. J. Marciano, Phys. Rev. D 52, R2619 (1995).

[14] A. Czarnecki, B. Krause, and W. J. Marciano, Phys. Rev. Lett. 76, 3267 (1996).
[15] A. Czarnecki, W. J. Marciano, and A. Vainshtein, Phys. Rev. D 67, 073006 (2003); 73, 119901(E) (2006).

[16] T. Gribouk and A. Czarnecki, Phys. Rev. D 72, 053016 (2005).

[17] C. Gnendiger, D. Stöckinger, and H. Stöckinger-Kim, Phys. Rev. D 88, 053005 (2013).

[18] S. Heinemeyer, D. Stockinger, and G. Weiglein, Nucl. Phys. B699, 103 (2004).

[19] P. Marquard, Nucl. Part. Phys. Proc. 260, 107 (2015).

[20] M. Tanabashi et al. (Particle Data Group), Phys. Rev. D 98, 030001 (2018).

[21] G. W. Bennett et al. (Muon g-2 Collaboration), Phys. Rev. Lett. 89, 101804 (2002); 89, 129903(E) (2002).

[22] G. W. Bennett et al. (Muon g-2 Collaboration), Phys. Rev. Lett. 92, 161802 (2004).

[23] G. W. Bennett et al. (Muon g-2 Collaboration), Phys. Rev. D 73, 072003 (2006).

[24] M. Davier, A. Hoecker, B. Malaescu, and Z. Zhang, Eur. Phys. J. C 71, 1515 (2011); 72, 1874(E) (2012).

[25] J. Prades, E. de Rafael, and A. Vainshtein, Adv. Ser. Dir. High Energy Phys. 20, 303 (2009).

[26] P. J. Mohr, B. N. Taylor, and D. B. Newell, Rev. Mod. Phys. 84, 1527 (2012).

[27] A. Chapelain (Muon g-2 Collaboration), EPJ Web Conf. 137, 08001 (2017). 
[28] M. Otani (E34), J. Phys. Soc. Jpn. Conf. Proc. 8, 025008 (2015).

[29] T. Ishikawa, N. Nakazawa, and Y. Yasui, J. Phys. Conf. Ser. 920, 012009 (2017).

[30] J. A. M. Vermaseren, arXiv:math-ph/0010025.

[31] E. de Doncker, F. Yuasa, and Y. Kurihara, J. Phys. Conf. Ser. 368, 012060 (2012).

[32] E. de Doncker, F. Yuasa, K. Kato, T. Ishikawa, J. Kapenga, and O. Olagbemi, Comput. Phys. Commun. 224, 164 (2018).

[33] K. I. Aoki, Z. Hioki, M. Konuma, R. Kawabe, and T. Muta, Prog. Theor. Phys. Suppl. 73, 1 (1982).

[34] J. Fujimoto, M. Igarashi, N. Nakazawa, Y. Shimizu, and K. Tobimatsu, Prog. Theor. Phys. Suppl. 100, 1 (1990).

[35] G. Belanger, F. Boudjema, J. Fujimoto, T. Ishikawa, T. Kaneko, K. Kato, and Y. Shimizu, Phys. Rep. 430, 117 (2006).

[36] T. Kaneko, Comput. Phys. Commun. 92, 127 (1995).

[37] P. Cvitanovic and T. Kinoshita, Phys. Rev. D 10, 3978 (1974).
[38] P. Cvitanovic and T. Kinoshita, Phys. Rev. D 10, 3991 (1974).

[39] C. G. Bollini and J. J. Giambiagi, Nuovo Cimento B 12, 20 (1972); https://link.springer.com/article/10.1007/ BF02895558.

[40] G. 't Hooft and M. J. G. Veltman, Nucl. Phys. B44, 189 (1972).

[41] N. Nakanishi, Quantum Field Theory (in Japanese) (Baihukan, Tokyo, 1975), p. 271.

[42] A. Sidi, Practical Extrapolation Method (Cambridge University Press, Cambridge, England, 2003).

[43] H. Takahashi and M. Mori, Publ. RIMS Kyoto Univ. 9, 721 (1974).

[44] K. Fujikawa, Phys. Rev. D 7, 393 (1973).

[45] S. D. Joglekar, Phys. Rev. D 10, 4095 (1974).

[46] K.-i. Shizuya, Nucl. Phys. B109, 397 (1976).

[47] A. Das, Phys. Rev. D 26, 2774 (1982).

[48] J. C. Romao and A. Barroso, Phys. Rev. D 35, 2836 (1987).

[49] F. Boudjema and E. Chopin, Z. Phys. C 73, 85 (1996). 